\title{
An Energy-Efficient Elevator Operating System that Considers Sensor Information and Electricity Price Changes in Smart Green Buildings
}

\author{
Hyunkyoung $\mathrm{Choi}^{1}$, Kyungwoon $\mathrm{Cho}^{2}$, Hyokyung Bahn ${ }^{1}$ \\ 1. Dept. of Computer Science \& Engineering, Ewha University, Seoul, Republic of Korea \\ 2. Embedded Software Research Center, Ewha University, Seoul, Republic of Korea \\ E-mail:bahn@ewha.ac.kr
}

Received: 5 November 2018; Accepted: 25 November 2018; Available online: 1 March 2019

\begin{abstract}
In modern smart buildings, the energy consumption of a building is monitored every time. Smart buildings are also equipped with sensors that can collect various physical data such as temperature, motion, and light. In this paper, we use smart sensor technologies in the design of an efficient elevator operating system (EOS). Specifically, multiple sensor devices are used together to detect elevator passengers' behavior before they arrive at the elevator door and press the elevator call button. The detected information is then delivered to EOS through building networks and the scheduling system utilizes this information for the efficient control of the elevator cars. Specifically, when the number of passengers becomes large, EOS increases the number of working elevator cars to reduce the waiting time of passengers. In contrast, when the elevator traffic lessens, EOS reduces the number of working elevator cars in order to save the energy consumption. Experimental results with a wide range of configurations show that our EOS outperforms the conventional elevator scheduling system that does not consider sensor information or electricity price changes.
\end{abstract}

Keywords: Vertical transportation; Indoor sensors; Smart building; Elevator operating system; Elevator.

\section{Introduction}

With the recent advances in indoor sensor technologies as well as the increasing diffusion of smart meters, various kinds of smart living services within modern buildings are being realized. In particular, with the enhancement of sensor technologies, various indoor contexts such as temperature, motion, and light can be instantly collected. This allows the detection and tracking of environmental conditions and human behaviors, which will be exploited for a plenty of services such as energy saving, comfort, healthcare and security [1,2]. HVAC (Heating, Ventilating, and Air Conditioning) is a representative system that utilizes sensor technologies. HVAC controls the air condition and temperature of a building according to the weather and the existence of human beings in the building. Elevator scheduling is another important service that can be involved in sensor technologies in a smart building. There is a report that HVAC and elevator service are the two major complaints of building tenants.

In this paper, we present a new elevator operating system (EOS) that utilizes sensor technologies in smart green buildings. As human behaviors and movements can be estimated precisely with modern indoor sensor technologies such as camera, audio, optical, and floor sensors as shown in Figure 1, EOS can recognize the arrival of passengers before actual call buttons are pressed. The system, then, utilizes this information for the efficient scheduling of elevator cars, leading to reduced latency and energy consumption. Though lots of researches on elevator scheduling have been performed to achieve the same goal, utilizing sensor technologies to obtain passenger information is in the initial stage of elevator scheduling problems. This paper uses multiple sensor technologies to predict passenger information more precisely; we validate the effectiveness of the prediction and present a general framework of the group elevator scheduling system utilizing it.

There are several performance criteria of elevator scheduling systems such as minimizing the waiting time, minimizing the riding time, and reducing the energy consumption. Most studies have focused on minimizing the average waiting time since passenger's dissatisfaction grows rapidly as the waiting time increases. Our elevator scheduling system focuses on reducing the energy consumption as well as minimizing the average waiting time. Minimizing both the energy consumption and the passengers' waiting time is not a simple issue as they sometimes conflict. For example, decreasing the number of working elevator cars will be helpful in saving the energy consumption, but it may increase the waiting time of passengers. Furthermore, because of the complex elevator dynamics, complicated traffic patterns, and multiple objectives to be optimized, state-of-the art studies use various optimization skills like genetic algorithms and fuzzy systems [4, 5]. 


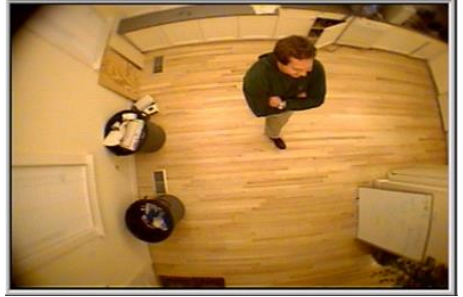

(a) Camera sensors

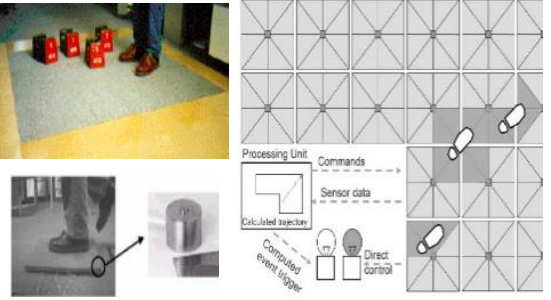

(b) Floor sensors

Fig. 1. Human tracking technologies with indoor sensors $[1,2]$

Some studies estimate elevator usage patterns by making use of the peak hour distribution analysis [5]. However, estimation and utilization that existing studies use is limited since elevator operating systems can be aware of the arrival of passengers only after they press the call buttons. Unlike existing studies, the proposed EOS recognizes the arrival of passengers with indoor sensor technologies before they actually press the call buttons, which is then used for efficient scheduling of elevator cars.

With respect to the waiting time criterion, it is important to guarantee the tolerable latency of each passenger. In general, passengers' dissatisfaction grows as their waiting latency increases, but there is a certain threshold that passengers are difficult to tolerate $[4,5,7]$. That is, when a passenger's waiting time is over this threshold, his/her dissatisfaction grows rapidly. Thus, our EOS is designed to keep the tolerable latency of passengers even though we need to pay more energy cost. Our EOS is adaptively controlled to support passengers' satisfaction as well as to minimize the energy cost. Specifically, when the number of passengers becomes large (i.e., heavy traffic), EOS increases the number of working elevator cars. This will eventually guarantee the tolerable latency of passengers. On the other hand, when the elevator traffic lessens, EOS reduces the number of working elevator cars. This will eventually save the energy cost.

To evaluate the performance of our EOS, we conduct extensive simulations. The results show that the proposed EOS performs better than the conventional elevator scheduling system in terms of the passengers' average waiting time, the maximum waiting time, and the energy consumption of the elevator system significantly.

\section{The proposed system}

In this section, we describe the details of the proposed system called EOS (Elevator Operating System). Our EOS is different from conventional elevator scheduling systems in that it adaptively controls elevator scheduling considering the passenger traffic monitored by indoor sensor technologies, thereby minimizing the energy consumption as well as the passenger's waiting time. To do so, we inject a learning module into the control unit of EOS, which monitors the change of passengers' traffic detected by sensors.

Figure 2 depicts the overall structure of EOS; it is composed of three units, namely the cost evaluation unit, the assignment unit, and the control unit. The cost evaluation unit evaluates the estimated cost of each elevator car to serve a request. The assignment unit determines the car to serve the request. The control unit keeps track of the passenger traffic with indoor sensors, and periodically adjusts the control parameter.

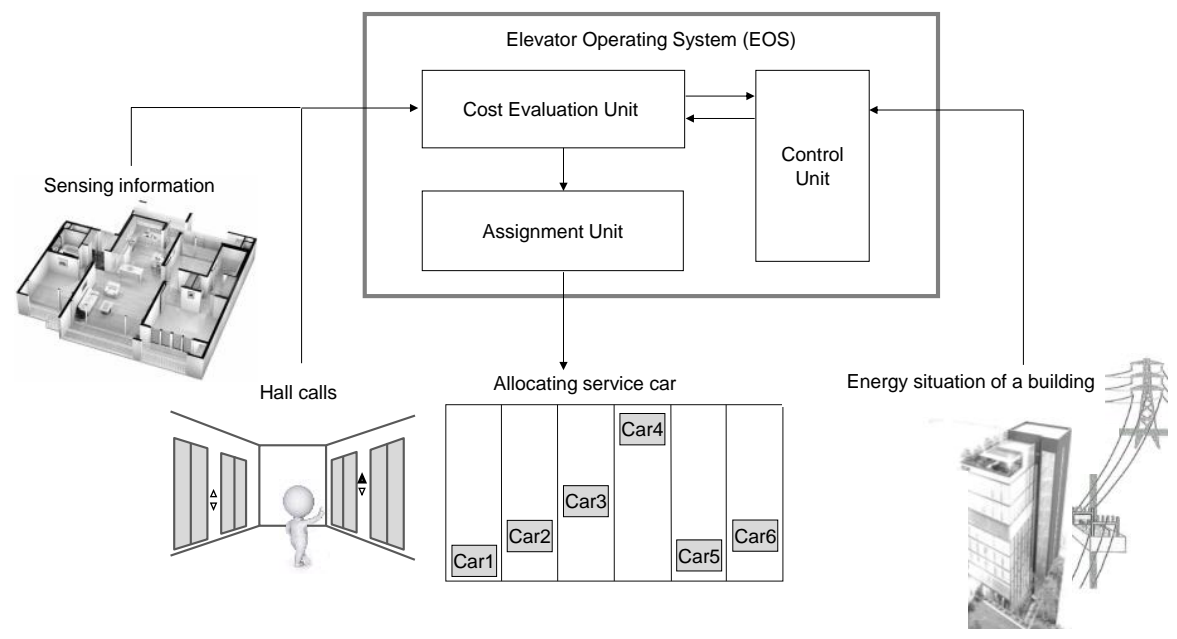

Fig. 2. Basic architecture of the proposed elevator operating system. 
In the elevator request interfaces, there are two types of call buttons: hall call and car call [9, 10]. A hall call is triggered when a passenger presses upward or downward button at hall in order to ride an elevator car. A car call is triggered when a passenger rides a car and presses the destination-floor button in the elevator car. As we use sensor technologies to estimate candidate passengers, there is another kind of call, a reservation call. A reservation call is issued when indoor sensors detect the arrival of candidate passengers and estimate the time when the passenger will reach the elevator; and generate a hall call based on the estimated time.

When a hall call occurs, the elevator scheduling system determines the car to serve that request. In our system, the cost evaluation unit calculates the estimated cost of each elevator car from its current location to the requested floor. We represent the cost as the weighted sum of the electricity charge and the expected waiting time. The expected waiting time $\operatorname{COST}_{\text {TIME }}$ of a car $w$ from the $m$-th floor to the $n$-th floor is computed as

$$
\begin{aligned}
& \operatorname{COST}_{\text {TIME }}(w, m, n)=\sum\left(T I M E_{M O V E}+T_{\left.M I M E_{S T O P}\right)}\right. \\
& T I M E_{M O V E}=\left\{\begin{array}{ll}
2 \sqrt{\frac{D I S T}{A S C}} & \text { if DIST<DIST } \\
2 \sqrt{\frac{D I S T_{M I N}}{A S C}}+\frac{D I S T-D I S T_{M I N}}{U S C} & \text { otherwise }
\end{array}\right\} \\
& T I M E_{S T O P}=T I M E_{O P E N}+T I M E_{B O A R D}+T I M E_{C L O S E}
\end{aligned}
$$

where DIST is the moving distance of the car from its current position to the next stop position, $D I S T_{M I N}$ is the minimum distance required for constant speed motion, $A S C$ is the constant acceleration of the car, and USC is the uniform velocity of the car. TIME $E_{O P E N}, T I M E_{B O A R D}$, and TIME $E_{C L O S E}$ represent the latency to open the elevator door, board the car, and close the elevator door, respectively. We set constant values for TIME $E_{O P E N}, T_{M} E_{B O A R D}$, and $T I M E_{C L O S E}$. If the car needs to visit multiple floors while moving from the $m$-th floor to the $n$-th floor, each $T I M E_{M O V E}$ and $T I M E_{S T O P}$ are added to $C O S T_{T I M E}$.

The energy cost $C O S T_{E N E R G Y}$ of the car $w$ from the $m$-th floor to the $n$-th floor is determined based on the movement of an elevator car as follows.

$$
\begin{aligned}
& \operatorname{COST}_{E N E R G Y}(w, m, n)=\left\{E_{W A K E U P}+\sum\left(E_{A C C E L}+E_{U N I}\right)\right\} \\
& E_{U N I}=\left|W_{C A R}+W_{P}-W_{C W T}\right| g h
\end{aligned}
$$

where $E_{A C C E L}$ and $E_{U N I}$ are energy consumptions in accelerating and constant speed motions respectively. $W_{C A R}$ is the mass of the car, $W_{P}$ is the sum of passengers' weight, $W_{C W T}$ is the weight of the counterweight, $g$ is the gravity constant, and $h$ is the distance between the current floor and the target floor. We set $E_{A C C E L}$ as a constant value as accelerating an elevator car is a short motion, which happens within a short period of 0.1 to 0.5 second [7]. If a car needs to visit multiple floors during its movement, each $E_{A C C E L}$ is accumulated.

After calculating all cars' cost with respect to the waiting time and the energy consumption, the cost evaluation unit sends the results to the assignment unit. Then, the assignment unit allocates the car that is expected to incur the minimum cost to this request.

The total cost function $\Phi(w, m, n)$ of a car $w$ from the $m$-th floor to the $n$-th floor is evaluated by the weighted sum of $\operatorname{COST}_{\text {TIME }}(w, m, n)$ and $\operatorname{COST}_{\text {ENERGY }}(w, m, n)$ as follows.

$$
\Phi(w, m, n)=\alpha \operatorname{COST}_{T I M E}(w, m, n)+(1-\alpha) \operatorname{COST}_{E N E R G Y}(w, m, n)
$$

where $\alpha(0 \leq \alpha \leq 1)$ is a weight parameter that decides how much emphasis is given to either the waiting time factor or the energy cost factor. The car with the least total cost is determined as the service car.

The cost evaluation unit, then, delivers the information to the control unit. Based on this information, the control unit monitors the state of the system, and periodically updates the control parameter $\alpha$. The initially value of the parameter $\alpha$ is set by the system administrator and is updated periodically based on the average waiting time of the latest interval in order to keep the passenger's tolerable latency. The threshold of the tolerable latency is set to the maximum round trip time (RTT) of an elevator car divided by the number of elevator cars [7]. When the passenger traffic becomes very high, the control unit increases the value of $\alpha$ to keep the tolerable latency threshold although it is not the administrator's intention. When the traffic returns to the normal state, the control unit decreases the value of $\alpha$ to save the energy consumption. Even though the passenger traffic becomes very light, the system sets $\alpha$ to the initial value and does not decrease any more in order to conform to the administrator's intention. 


\subsection{Sensor-based detection}

To assess the effectiveness of multiple sensor devices in detecting passenger information, we equipped RFID, video, and floor sensors in a twenty-story building for residence, and collected sensed data for enrolled users during a day. The building authenticates the enrolled users at the gate of the underground parking area or the main entrance of the ground floor using an RFID tag or a password. We first collect sensor data at this time to make a reservation call. Less than 120 seconds are needed for a candidate passenger to move from this location to the elevator door. Along the hallway towards the elevator, floor sensors are located, and they can detect the moving direction of people through the path of the step. This information is collected about 60 seconds prior to the actual hall call at the elevator. Video sensors are also located at the ceiling of the hallway, and they can also recognize the movement of people. The distance from this location to the elevator is about 30 seconds or less. We set the default moving direction of reservation calls to "up" at the parking area and the ground floor. Note that a reservation call is dropped from the request queue when it is not continued to appear in the subsequent sensors. For example, when a reservation call is issued 120 seconds prior to the actual hall call through RFID tags but there is no corresponding reservation call through video or floor sensor, the request is dropped from the scheduling queue.

In the residence area ranging from the 2nd to 20th floors, each home has its front door and there are floor sensors along the hallway. 60 seconds or so are needed to move from the front door to the elevator including the locking time of the door. Video sensors are also located at the ceiling of the hallway, and the distance to the elevator is about 30 seconds or less. We set the default moving direction of reservation calls to "down" at residence floors.

We gathered the sensor data during a day and compared the reservation calls issued by sensor information and actual hall calls from passengers. We use a CCTV in front of the elevator car to collect the actual hall call information. The comparison result showed that more than $80 \%$ of the reservation calls eventually invoke actual calls. As reservation calls are dropped from the request queue if subsequent actual calls do not appear, the effect of false reservation calls is not significant. Note that our scheduler waits only for a certain limited time for reservation calls, and it promptly reacts to the changed request information. On the other hand, most requests benefit from these reservation calls by shortening their waiting time.

\subsection{Elevator scheduling systems}

There have been a variety of studies on the scheduling of elevator systems to reduce passengers' waiting time. Most of them focus on the group elevator systems, in which a single control unit schedules multiple elevators simultaneously. In group elevator systems, the scheduling problem becomes even more complicated, and thus various optimization techniques such as fuzzy systems, genetic algorithms, genetic network programming, and artificial neural networks have been adopted.

Igarashi et al. present a fuzzy system based group elevator scheduling systems [8]. When a passenger pushes the elevator call button, the system evaluates each elevator car using a fuzzy function and assigns the car with the largest evaluation value that can minimize the waiting time of the request. Kim et al. use genetic algorithms to optimize the scheduling of group elevator systems [6]. They employ cameras to detect the number of waiting passengers at each floor and assign multiple elevator cars simultaneously if the number of waiting passengers exceeds the capacity of a single elevator.

If an elevator scheduling system recognizes the destination floor of the passengers before they ride the car, the system could schedule elevators more efficiently. A conventional elevator system, however, has only two buttons (upward and downward) at each floor, and thus it cannot predict passengers' destination in advance. To address this problem, Amano et al. present an elevator system that has the destination-floor buttons at each floor. When a passenger intends to ride an elevator, he/she pushes a specific destination-floor button at the floor. This provides more information to the elevator operating system than just up and down buttons [11].

Mitsubishi Electric Corporation developed an RFID-enabled elevator system. The RFID tag informs the arrival of passengers and their destination, which are used for the elevator scheduling and security. By combining RFID and cameras, their system discerns if a person wants to use an elevator or is just walking near an elevator.

In most elevator environments, traffic patterns change periodically within a day, and a number of studies have presented traffic pattern based elevator operating systems. Pepyne and Cassandras analyzed up-peak traffic patterns of elevator systems and exploit the pattern in the elevator scheduling [12]. Brand et al. consider both uppeak and down-peak traffics [5]. Their system dispatches an empty elevator to a desired parking location before requests from users. The location is determined by the analysis of up-peak and down-peak traffic patterns. These parking strategies could minimize passengers' waiting time in common cases. The traffic of an elevator system changes continuously as time progresses. There have been researches on detecting and modeling these traffic changes. In such systems, EOS detects the current flow and chooses a scheduling policy among a set of readyprepared policies. Pepyne et al. present an elevator scheduling strategy that delays the elevator moving until the number of passengers inside it reaches a certain threshold [12]. 
Some recent studies consider the energy consumption of the elevator system as well as the passengers' waiting time. Since reducing the energy consumption and the passengers' waiting time sometimes conflict, optimization of these multiple goals becomes an even more complex problem. Kim et al. use fuzzy systems to solve this problem [10]. Lee et al. use a weighted sum technique to satisfy the two goals and periodically adjust the weight value [4].

Recently, smart building prototypes are developed that contain various home sensors such as video sensors, floor sensors, and small battery-powered wireless devices. We can obtain plenty of context information from these sensors and the information can be exploited for smart home services. Kidd et al. present the aware home prototype [13]. It consists of several living spaces such as bedroom, bathroom, living room, dining room, kitchen, laundry room, and office. In this prototype room, video sensors are used to trace human locations. A person in this home is tracked and his/her activities are reported on the map. With this information, human behaviors can be analyzed and predicted.

Orr et al. present the smart floor for user identification and tracking [2]. They have created a system for identifying people according to their footstep force profile. Because people interact with the smart floor system, the location of the people can be traced and also be predicted. Addlesee et al. present the active floor, which is similar to the smart floor [3]. The active floor is the square grid of conventional tiles that is supported at the corners by cylindrical load cells which send weight changes of about 50 grams to the location system. With this information, the system can predict the future locations of people. Similarly, some research uses an array of capacitive sensors embedded in floor carpet to track the movement across a large area.

\section{Experimental results}

In this section, we present the performance evaluation results to assess the effectiveness of the proposed scheduling system. We perform experiments under two workload conditions. They consist of homogeneous traffic and non-homogeneous traffic. Table 1 lists the experimental configurations used in our experiments. The number of floors is 20 and the number of cars is 6, respectively. The maximum capacity of an elevator car is 20 passengers. The traffic of passengers is generated by the Poisson process with the average arrival rates of 5 to 50 passengers/minutes similar to previous studies [6].

Table 1. Simulation configurations

\begin{tabular}{l|l}
\hline Parameter & Value \\
\hline \# of floors & 20 \\
\# of cars & 6 \\
Capacity of a car & 20 people/car \\
Uniform velocity & $3 \mathrm{~m} / \mathrm{sec}$ \\
Acceleration & $1 \mathrm{~m} / \mathrm{sec}^{2}$ \\
Height of a floor & $3 \mathrm{~m}$ \\
Open and close latency & $2 \mathrm{sec}$ \\
Boarding latency & $1.5 \mathrm{sec} /$ passenger \\
\hline
\end{tabular}

We compare our elevator scheduling system called Sensor-aware Elevator Scheduling (SES) with a conventional elevator system (CS) that does not consider the sensor information. According to the prototype settings in Section 2.1, we perform three SES configurations: SES-G-30, SES-G-60, and SES-G-120. In SES-G30 , the reservation call is issued 30 seconds before passengers make the actual hall call. Similarly, SES-G-60 and SES-G-120 issue the reservation calls 60 and 120 seconds before actual hall calls, respectively.

Figure 3 shows the average waiting time of CS and SES groups (SES-G-30, SES-G-60 and SES-G-120) as the arrival rate varies. As shown in the figure, SES-G performs consistently better than CS irrespective of the arrival rate. Specifically, the average waiting time of SES-G-30 is better than CS by $22 \%$ on average and up to $30 \%$. The performance gain of SES-G-120 is relatively small because the car picks up many passengers at a time. Figure 4 shows the total amount of energy consumed during the operation of the elevator system. SES groups again perform better than CS. Among the three configurations, SES-G-120 performs the best as the car picks up more people at a single round trip time than other elevator scheduling systems. The performance improvement of SES-G-120 against CS is as much as $29 \%$ on average and up to $31 \%$ in terms of the energy consumption.

To reflect the real situation of elevator systems more precisely, we simulate the case of non-homogeneous arrival rate workloads with up-peak and down-peak traffics. 


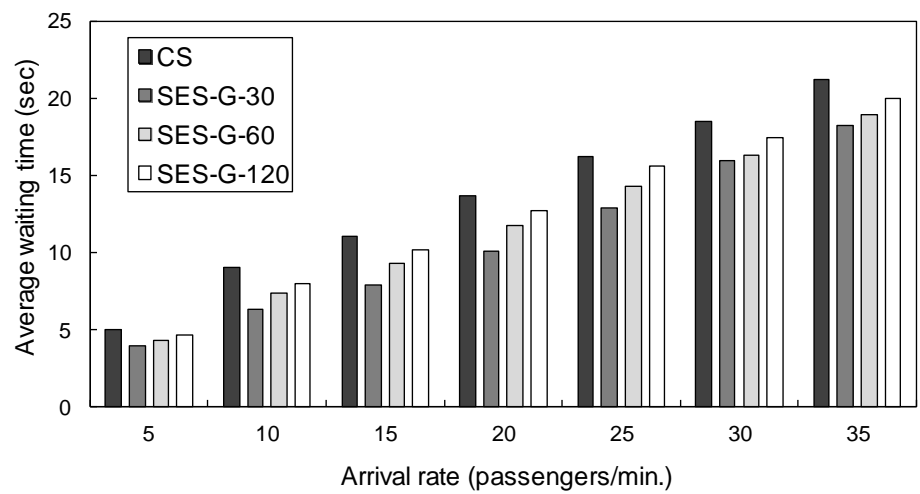

Fig. 3. Passenger's waiting time as a function of the arrival rate.

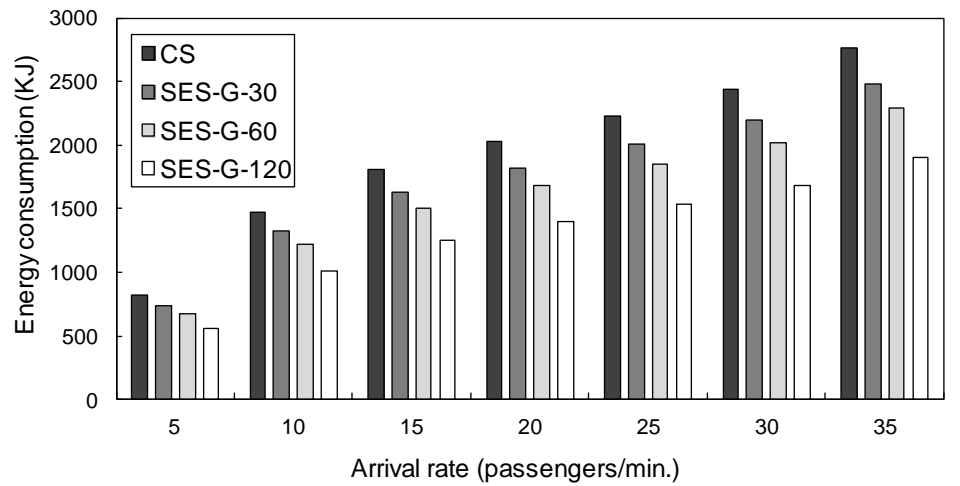

Fig. 4 Energy consumption as a function of the arrival rate.

Figure 5 shows the results for non-homogeneous traffic patterns. Again, SES-G performs better than CS for all performance measures. As shown in Figure 5(a), the average waiting time of SES-G-30 is $16 \%$ better than CS. Similar to the results in Figure 3, the performance gain of SES-120 is relatively small in terms of the average waiting time. However, this is not the case for the maximum waiting time as depicted in Figure 5(b). Irrespective of SES configurations, the improvement of the maximum waiting time is as much as $7 \%$ on average. Figure 5(c) shows the total amount of energy consumed during the operation of the elevator system. Similar to the homogeneous traffic cases, SES-120 performs the best; the improvement is $31 \%$ compared to CS in terms of the energy consumption.

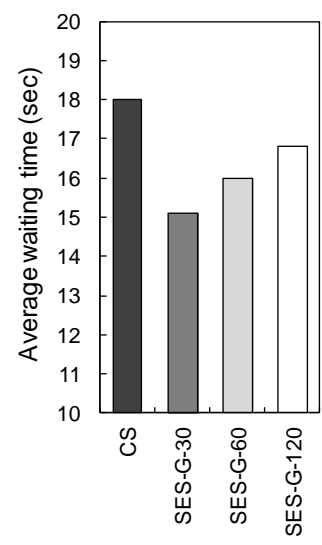

(a) Average waiting time

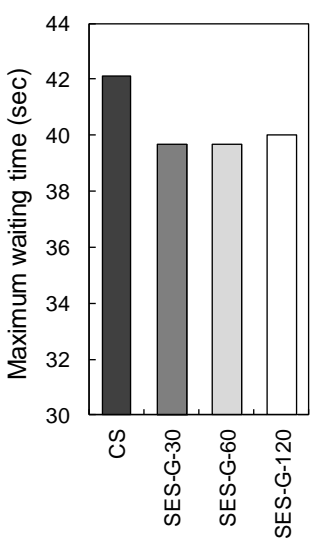

(b) Maximum waiting time

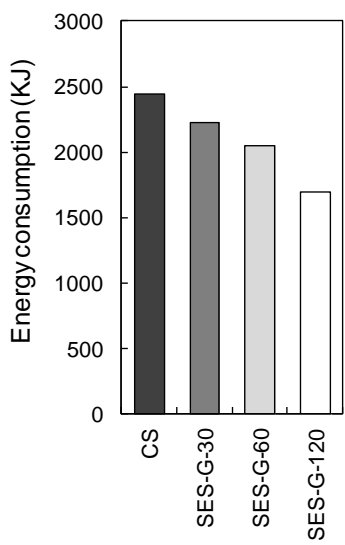

(c) Energy consumption

Fig. 5. Results with non-homogeneous workload conditions. 


\section{Conclusions}

As smart grid and indoor sensor technologies are advancing, various smart living services are being realized. This paper proposed a new elevator operating system that utilizes indoor sensor technologies in smart green buildings. Specifically, we use multiple sensor devices to detect passengers' information before they actually push the elevator call button. In particular, three types of sensor devices, RFID, video, and floor sensors are used to estimate passenger information precisely. By utilizing this prior information, our elevator operating system controls multiple elevator cars more efficiently than conventional on-demand elevator schedulers. To validate the efficiency of the proposed system, we conducted experiments under various workload configurations including homogeneous and non-homogeneous traffic workloads. Our simulation results showed that the proposed elevator operating system significantly reduces the average waiting time, the maximum waiting time, and the energy consumption.

\section{Acknowledgment}

This paper was supported by the ICT R\&D program of MSIP/IITP (2018-0-00549, Extremely scalable orderpreserving OS for manycore and non-volatile memory). Hyokyung Bahn is the corresponding author of this paper.

\section{References}

[1] Essa IA. Ubiquitous sensing for smart and aware environments. IEEE personal communications. 2000;7(5):47-49.

[2] Orr RJ, Abowd GD. The smart floor: A mechanism for natural user identification and tracking. In: Proc. Int. Conf. Human Factors in Computing Systems (The Hague, Netherlands). ACM;2000. p. 275-276.

[3] Addlesee M, Jones A, Livesey F, Samaria F. The ORL active floor. IEEE Personal Communications $1997 ; 4: 35-41$.

[4] Lee S, Bahn H. An energy-aware elevator group control system. In: Proc. 3rd IEEE International Conference on Industrial Informatics; 2005. p.639-643.

[5] Brand M, Nikovski D. Optimal parking in group elevator control. In: Proc. IEEE International Conference on Robotics \& Automation; 2004. p.1002-1008.

[6] Kim JH, Moon BR. Adaptive elevator group control with cameras. IEEE Transactions on Industrial Electronics. 2001;48(2):377-382.

[7] Koh KS. Introduction to Elevator System. Seiwunsa. 1990.

[8] Igarashi K, Take S, Ishikawa T. Supervisory control for elevator group with fuzzy expert system. In: Proc. IEEE International Conference Industrial Technology; 1994. p. 133-137.

[9] A. Fujino, T. Tobita, and K. Yoneda, An on-line tuning method for multi-objective control of elevator group, Proc. IEEE Industrial Electronics, Control, Instrumentation, and Automation; 1992. p.795-800.

[10] Kim C, Seong KA, Lee-Kwang H, Kim JO. Design and implementation of a fuzzy elevator group control system. IEEE Transactions on Systems, Man, and Cybernetics-Part A: Systems and Humans. 1998;28(3):277287.

[11] Amano M, Yamazaki M, Ikejima H. The latest elevator group supervisory control system. Mitsubishi Elect. ADVANCE. 1996;67(1):88-95.

[12] Pepyne D, Cassandras C. Optimal dispatching control for elevator systems during uppeak traffic. IEEE Trans. Control Systems Technology 1997;5:629-643.

[13] Kidd CD, Orr RJ, Abowd GD, Atkeson CG, Essa IA, MacIntyre B, Mynatt E, Starner TE, Newstetter W. The aware home: a living laboratory for ubiquitous computing research. Lecture Notes in Computer Science 1999;1670:191-198.

(C) 2019 by the author(s). This work is licensed under a Creative Commons Attribution 4.0 International License (http://creativecommons.org/licenses/by/4.0/). Authors retain copyright of their work, with first publication rights granted to Tech Reviews Ltd. 